\title{
Scene Boundary Detection from Movie Dialogue: A Genetic Algorithm Approach
}

\author{
Amitava Kundu, Dipankar Das, and Sivaji Bandyopadhyay
}

\begin{abstract}
Movie scripts are a rich textual resource that can be tapped for movie content analysis. This article describes a mechanism for fragmenting a sequence of movie script dialogue into scene-wise groups. In other words, it attempts to locate scene transitions using information acquired from a sequence of dialogue units. We collect movie scripts from a web archive. Thereafter, we preprocess them to develop a resource of dialogues. We feed the dialogue sequence from a script to a Genetic Algorithm (GA) framework. The system fragments the sequence into adjacent groups of dialogue units or output 'scenes'. We use SentiWordnet scores and Wordnet distance for dialogue units to optimize this grouping so that adjacent scenes are semantically most dissimilar. Then we compare the resulting fragmented dialogue sequence with the original scene-wise alignment of dialogue in the script.
\end{abstract}

Index Terms-Dialogue, genetic algorithm, movie script, scene.

\section{INTRODUCTION}

$\mathrm{W}$ ITH loads of digital movies being consumed online, movie search has become an important concern in the information retrieval domain. A lot of studies have reported extraction of semantically meaningful information from movie data [1-4]. Such information is useful in browsing, searching, and genre classification. Feature-length movies usually have extensive lengths. This makes movie information search with sole aid of audio-visual data a daunting task. Therefore, movie search mostly relies on textual metadata associated with movies such as title, keywords, and plot.

In general, a movie includes certain textual information such as subtitles and annotations. In addition, movie scripts provide a wealth of information about the dialogue, characters, story, setting and action in movies. They are instrumental in bringing the filmmaker's imagination to life. Most of the movie scripts conform to a set of semi-regular formatting conventions (www.screenwriting.info). These standard terms and conventions can be reaped in order to extract useful elements from scripts.

Scene boundary detection is a fundamental research area

Manuscript received December 15, 2012. Manuscript accepted for publication January 11, 2013.

Amitava Kundu and Sivaji Bandyopadhyay are with the Department of Computer Science \& Engineering, Jadavpur University, Kolkata-700032, India (e-mail: amitava.jucse@gmail.com, sivaji_ju_cse@yahoo.com).

Dipankar Das is with the Department of Computer Science \& Engineering, National Institue of Technology, Meghalaya, Laitumkhrah, Shillong-793003, Meghalaya, India (email: dipankar.dipnil2005@gmail.com). which allows navigating movies with semantic ways. A movie consists of a thousand of shots and hundreds of thousands of frames. A scene is a set of shots and a boundary of one of these shots becomes a scene boundary. A number of studies have attempted scene boundary detection that mainly analyzes visual features and group shots according to the visual shot similarity [5], [6]. Scene boundary detection has also been attempted with the aid of scripts and subtitles. The scriptsubtitles alignment mechanism has been used in [7-9] for the purpose. It may be mentioned in this context that a script, unlike its subtitle counterpart does not convey any information about the time period in the movie.

Crafted aspects of movie dialogue are useful as they are authored deliberately to convey feelings and perceptions of the characters portrayed [2], [3]. Additionally, the screenplay specifies the emotion of utterances with psychological state descriptors. The current article describes a method of detecting scene boundaries/transitions from movie script dialogues. For the purpose, we have prepared textual resource containing structured information from movie scripts. Scripts available online have been utilized for the purpose. For the experiments, each script has been considered a sequence of dialogue units only. Then an attempt has been made to group the dialogue units scene-wise, as they would have appeared in the movie.

Our method segments the sequential dialogue units based on their changing semantic content and affect. It fragments the sequence of dialogue units in such a fashion that consecutive groups/clusters of dialogue text are most 'dissimilar', semantically and affect-wise. A scriptwriter frames his scenes carefully to narrate his story as per his intentions. We have assumed that each scene he writes is a coherent entity. Since we have chosen to use dialogue only, our notion of a scene for this task has been that a 'scene' is a dialogue sequence which sticks together semantically and sentiment-wise and it is somewhat different in the same sense from its neighboring 'scenes'. We have formulated this task as an optimization problem and adopted a Genetic Algorithm (GA) approach to this head. Genetic Algorithms (GAs) are a class of adaptive, randomized search and optimization techniques guided by principles of natural evolutions and genetics [10], [11]. This being fundamentally an optimized segmentation task, GA seemed to be most appropriate for our purpose.

The rest of the paper is structured as follows. Section II provides the rudiments of movie scripts and a detailed description of resource preparation. Section III describes in 
detail how Genetic Algorithm has been incorporated in our experiments. Section IV provides the results and observations. Finally, we summarize and present the future directions in Section V.

\section{RESOURCE PREPARATION}

Most of the feature-length films are produced with the aid of scripts [12] or screenplays. The script presents a detailed vision of the story, settings, dialogues, situations and actions of a film. It gives the filmmakers, the actors and the crews the necessary assistance in bringing the intended situation to life. Thus it can be looked upon as a rich resource that provides vivid textual descriptions of semantic objects within a film.

The Internet Movie Script Database (IMSDb; www.imsdb. com) is a huge online repository of movie scripts. Hundreds of movie scripts, coming straight from the filmmakers, are available for download. In the present task, scripts were collected from the above mentioned database but the collected scripts were subjected to some necessary preprocessing where the scenes were separately identified and stored in a structured format. The following subsection provides a general overview of the elements in a film script followed by the details of preprocessing steps.

\section{A. Highlights of a Movie Script}

As mentioned above, a movie script delineates the story, character, setting, action and dialogue of a film [9], [12]. Additionally, the camera directions and shot boundaries are also included. The script may go through a number of revisions prior to production of the movie. Often, after all the shots have been edited the resulting movie may not conform to all the minute details of the original script.

In general, the actual content of a script follows a semiregular format as shown in Figure 1, which shows a snippet of script from the movie Godfather-II. In a script, a scene starts with the slug line which establishes the physical context of the action that follows. It indicates whether a scene takes place inside or outside (INT or EXT), the name of the location (e.g. 'THE CHURCH PLAZA'), and can potentially specify the time of day (e.g. DAY or NIGHT). Important people and key objects are usually highlighted by capitalizing their names.

The bulk of a script is comprised of dialogue description. Actions of characters are described along with the dialogues for each character. Dialogues have the character name (usually in all caps) followed by the dialogue text. An optional (V.O.) or (O.S.) may follow the character name indicating that the speaker should be off-screen (V.O. stands for a "Voice Over"). In most scripts, dialogue actions are clearly separated from action and camera directions by enclosing the non-dialogue descriptions in brackets. Usually scarce, parenthetical directives convey phenomena such as inflection or mood. An example of a dialogue unit follows:

GARDNER: (a little nervous) I've heard a lot about you, Mr. Corleone.

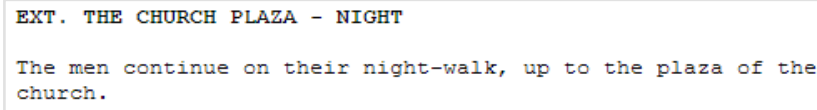

Fig. 1. Portion of a screenplay of the film Godfather-II

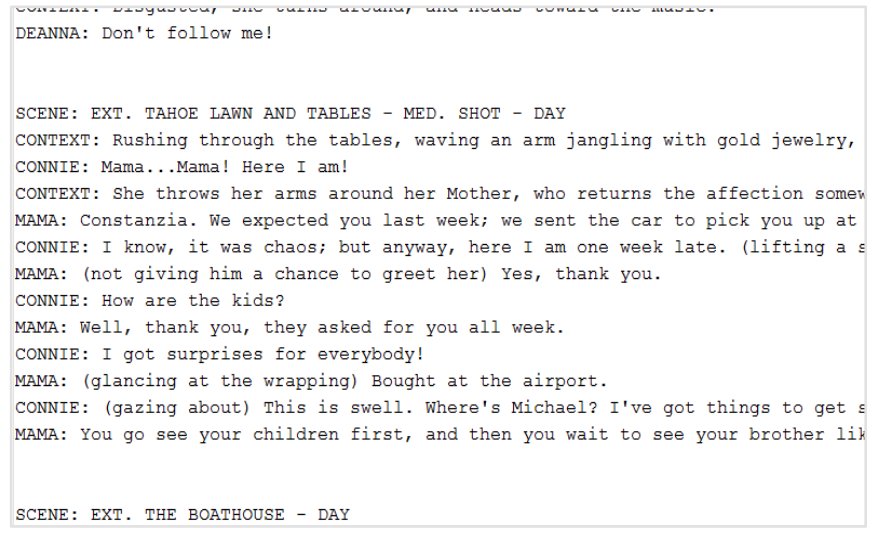

Fig. 2. Snippets of a resource file showing a typical scene from Godfather-II screenplay

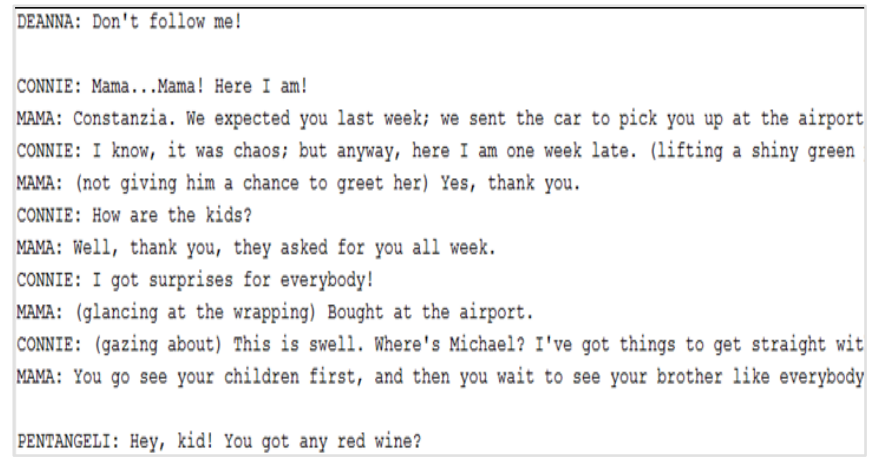

Fig. 3. Snippets of a resource file showing the dialogues of the scene from Godfather-II shown in Fig. 2.

\section{B. Preprocessing of scripts}

As mentioned already, movie scripts are written in a semiregular format. Variety of formats makes preprocessing a challenging task. To accomplish our experiments, we needed to prepare a structured resource from scripts so that we could evaluate the success of our scene boundary detection algorithm later on. Using knowledge of the usual anatomy of movie scripts outlined in the above subsection, the 
information pertaining to different scenes has been identified. In each of the sets of resource files, one for each script, the scenes have been stored in a structured format, as indicated in Figure 2. A scene sets off with the scene heading and the following line describes either a dialogue or an action. Each dialogue turn starts with the name of the speaker in capitals followed by the text. An action starts off with the word "CONTEXT" and describes what is going on in the scene. Each scene is separated from the following and the preceding ones by blank lines. A significant amount of manual labor has been invested in making necessary refinements and corrections before storing the resource in the required structured format.

Additionally, another set of resource files where scene-wise dialogues are stored have been prepared. Here dialogues from different scenes are separated with blank lines. All context information and scene headings have been excluded. These files have been prepared so that a script can later on be considered as a sequence of dialogue units without any knowledge of scene boundary (see Figure 3). However, scenes without dialogue were also abundant. They have been thus ignored altogether.

\section{SYSTEM FRAMEWORK}

In the experimentation, each script has been considered merely as a sequence of dialogue units excluding the context information (and even parenthesized directives).

Our objective was to detect the scene transitions looking at a sequence of dialogue texts. A close manual examination revealed abrupt changes in overall topic and affect across dialogues from consecutive scenes in many cases. This observation motivated us to take an attempt for detecting scene transitions. We have incorporated a genetic algorithm approach for the purpose. Figure 4 and Figure 5 illustrate the scheme and convey the objective.

\section{A. The Genetic Algorithm Approach}

Genetic Algorithm (GA) is a search heuristic that mimics the process of natural evolution. GAs belong to the larger class of Evolutionary Algorithms (EA), which generate solutions to optimization problems using techniques inspired by natural evolution: crossover, mutation and selection.

In the present work, the challenge is to frame the task of detecting scene boundaries as an optimization problem. As mentioned above, the changes in topic or overall affect of dialogues have often been observed across consecutive scenes. Therefore, we have made a principal assumption that dialogues from consecutive scenes are "dissimilar" in some sense. The main idea is to segment the sequence of dialogue units into scenes in such a way that makes the consecutive dialogue groups as "dissimilar" as possible. In order to implement GA, we have utilized the Java Genetic Algorithms and Programming Package (JGAP version 3.6.2), an opensource Java based tool.

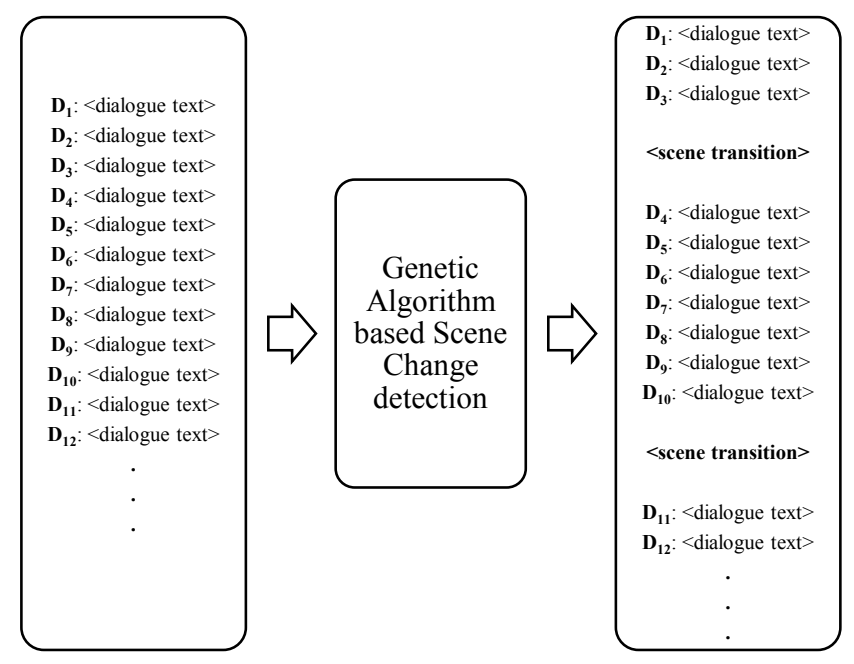

Fig. 4. The basic objective of our scheme. The input is a sequence of dialogue text as spoken in sequence by characters in film; $D_{1}, D_{2}, D_{3} \ldots$ The system outputs a scene-wise grouping of dialogue units. A group can be seen as set of dialogue units belonging to a particular scene

\section{1) Encoding.}

As a genetic algorithm applies to encoded candidate solutions or chromosomes, before one can proceed, an encoding technique of chromosomes has to be chalked out. We have devised a binary encoding technique for the purpose where a chromosome is a string of ' 0 's and ' 1 's. As already mentioned, we have treated a script merely as a sequence of dialogue units. If we consider a script with $n$ dialogue units, in our scheme, each chromosome is a binary string of length n- 1 . The encoding is such that a ' 1 ' at $\mathrm{m}^{\text {th }}$ allele position of a chromosome indicates a scene transition after the $\mathrm{m}^{\text {th }}$ dialogue unit while a ' 0 ' indicates scene continuation. Since the nth dialogue unit would be the last one in the script, encoding a transition for it in the solution string would be unnecessary. Hence the length of such a string/chromosome is n-1. Figure 6 illustrates the scheme vividly.

\section{2) Crossover, Mutation and Selection.}

Since binary encoding has been adopted, usual genetic operators have been conveniently deployed. Single point crossover has been adopted. Mutation involves bit flipping where a ' 0 ' is replaced by a ' 1 ' and vice-versa. Standard Roulette Wheel Selection has been applied.

\section{3) Population Size and Evolution.}

We have incorporated the elitist model of GA wherein the fittest chromosome from each generation is preserved so as to ensure the best solution is not lost. Population size has been kept 50. We run the GA up to 100 generations.

\section{4) Fitness Computation.}

The fitness function is at the heart of our genetic algorithm framework. Dialogues across consecutive scenes have been assumed to be different or dissimilar. 


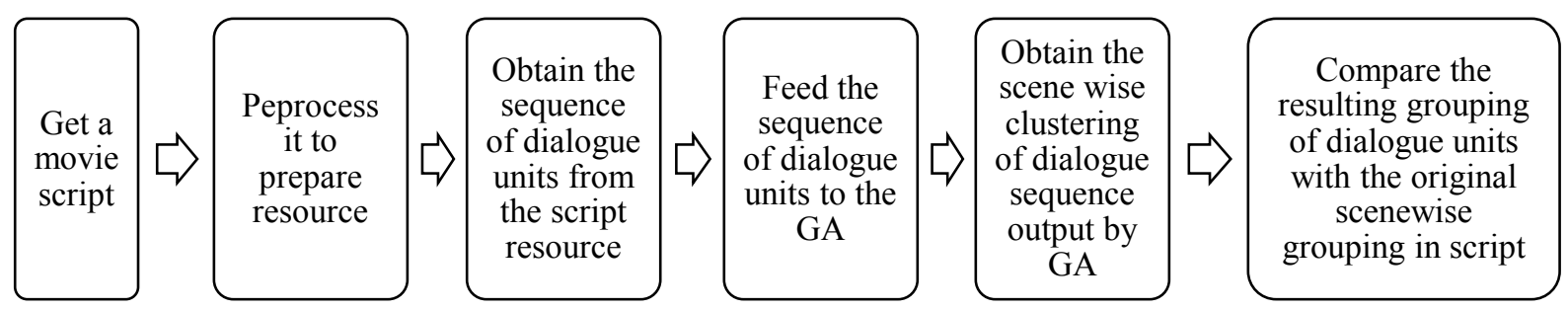

Fig. 5.Workflow of our system

In our scheme, those solutions that encode scene transitions resulting in greater dissimilarity across consecutive scenes have been considered as fitter chromosomes. Thus, given the binary encoding of each chromosome, the resulting scenesegmentation of dialogue units has been easily reconstructed. Thereafter, dissimilarity across consecutive groups of dialogue has been capitalized in computation of fitness. The SentiWordnet 3.0 [13] scores and Wordnet similarity of encoded scenes have also been accounted for.

\section{(a) SentiWornet scores.}

For every script, the Stanford CoreNLP ${ }^{1}$ (version 1.3.3) has been used to tokenize dialogue unit text, identify the named entities in turns and assign part-of-speech (POS) tags to tokens in a turn. Thereafter SentiWordnet 3.0 has been used to compute the total sentiment scores $^{2}$ of each dialogue unit. Sentiment scores of nouns excluding named entities, adjectives, adverbs and verbs only have been considered for this computation.

Consider the dialogue unit given below,

DEANNA: Relax, Freddie honey. Come dance with me.

The POS tagged version of the above with sentiment values indicated in parentheses is as follows,

\section{Relax $^{1} / \mathbf{V B}(-0.06668), /$, Freddie/NNPhoney ${ }^{2} / \mathbf{N N}(0.04166)$ ./. Come $^{3} / \mathbf{V B}(0.00529)$ dance $^{4} / \mathbf{N N}(0.0)$ with/IN me/PRP ./.}

Likewise, sum total sentiment score of all dialogues in every scene was computed, as encoded in any given chromosome. If a chromosome encoded scenes (groups of dialogue) $S_{1}, S_{2} \ldots S_{n}$ with total sentiment values $P_{1}, P_{2}, \ldots, P_{n}$ respectively, its fitness $F_{\text {sentiment }}$ is computed as follows,

$$
F_{\text {sentiment }}=\sum_{i=2}^{n}\left(P_{i}-P_{i-1}\right)^{2}
$$

\section{(b) Wordnet distance of keywords.}

Each dialogue unit has been tokenized, named entities have been identified and POS tags have been assigned to tokens using Stanford CoreNLP.

\footnotetext{
1 http://nlp.stanford.edu/software/corenlp.shtml

2 Online resource: http://sentiwordnet.isti.cnr.it/code/SWN3.java
}

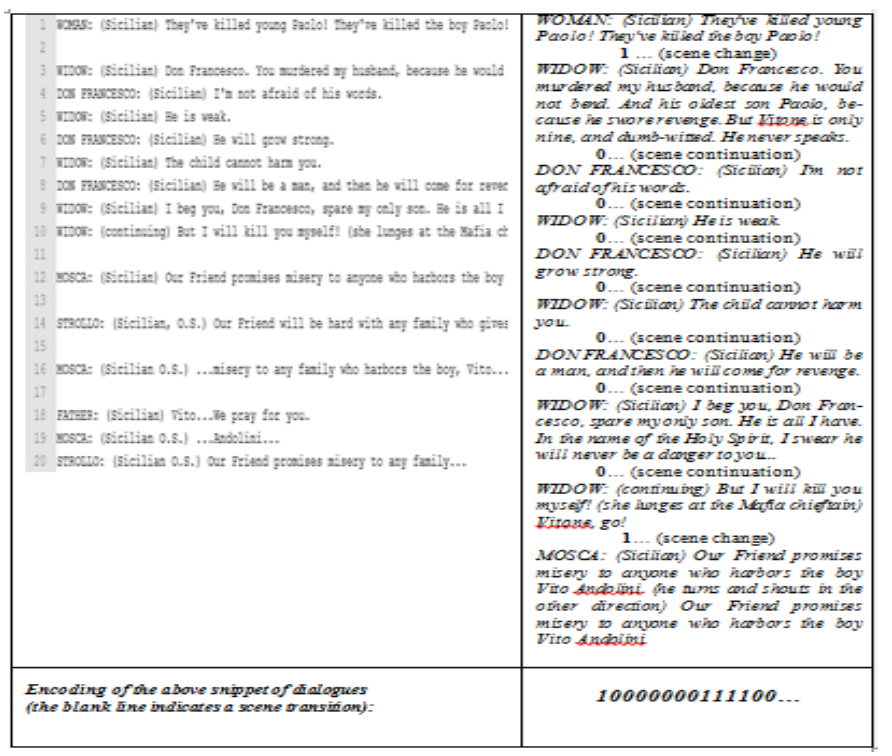

Fig. 6.Snippet of dialogue sequence from Godfather-II and its encoding.

Additionally, each of the dialogue units has been annotated with the CoreNLP parse annotator so as to obtain the Stanford semantic dependency relations [14] of each turn. Object relations have also been identified (if present) in each turn namely $d o b j$, iobj and pobj. Thereafter, the dependent components in those relations have been collected. A dependent in such an object relation has been considered as a potential keyword or topic word of the piece of dialogue. However, a dependent is ignored if it appeared in a list of stop words. An example with the keywords thus identified is given below (numbered ones are the identified words in question)

\section{CONNIE: I got surprises ${ }^{1}$ for everybody ${ }^{2}$ !}

Wordnet distance between keywords across consecutive dialogue groups have been considered for fitness computation. The getDistance() method of RiTaWordnet ${ }^{3}$ library has been deployed for computation of semantic distance between two words in Wordnet hierarchy.

Say the distance of two word is given by $d\left(w_{1}, w_{2}\right)$. It is the absolute value of the aforementioned distance metric between two words $w_{1}$ and $w_{2}$ in the Wordnet ontology.

\footnotetext{
${ }^{3} \mathrm{http} / /$ rednoise.org/rita/wordnet/documentation/index.htm
} 
TABLE 1.

PERFORMANCE OF SCENE BOUNDARY DETECTION

\begin{tabular}{|c|c|c|c|c|c|c|c|c|c|c|}
\hline \multirow[t]{2}{*}{ Movie } & \multirow{2}{*}{$\begin{array}{l}\text { \#Dialogue } \\
\text { Units in } \\
\text { Script }\end{array}$} & \multirow{2}{*}{$\begin{array}{l}\text { Actual \#scene } \\
\text { transitions in } \\
\text { script dialogue } \\
\text { sequence }\end{array}$} & \multicolumn{2}{|c|}{ With $F_{\text {baseline }}$} & \multicolumn{2}{|c|}{ With $\mathrm{F}_{\text {sentiment }}$} & \multicolumn{2}{|c|}{ With $\mathrm{F}_{\text {wordnet }}$} & \multicolumn{2}{|c|}{ With $\mathrm{F}_{\text {combined }}$} \\
\hline & & & $\mathrm{P}_{\mathrm{GA}}$ & $\begin{array}{c}\text { Transitions } \\
\text { correctly } \\
\text { detected }\end{array}$ & $\mathrm{P}_{\mathrm{GA}}$ & $\begin{array}{c}\text { Transitions } \\
\text { correctly } \\
\text { detected }\end{array}$ & $\mathrm{P}_{\mathrm{GA}}$ & $\begin{array}{c}\text { Transitions } \\
\text { correctly } \\
\text { detected }\end{array}$ & $\mathrm{P}_{\mathrm{GA}}$ & $\begin{array}{c}\text { Transitions } \\
\text { correctly } \\
\text { detected }\end{array}$ \\
\hline Godfather-II & 1190 & 195 & $31.2 \%$ & $69.3 \%$ & $51.3 \%$ & $46.7 \%$ & $57.6 \%$ & $47.1 \%$ & $55.6 \%$ & $48.7 \%$ \\
\hline Casino & 2478 & 452 & $41.7 \%$ & $64.4 \%$ & $52.3 \%$ & $49.1 \%$ & $51.7 \%$ & $42.7 \%$ & $52.7 \%$ & $49.6 \%$ \\
\hline \multirow[t]{2}{*}{ Citizen Kane } & 1014 & 88 & $37.8 \%$ & $73.3 \%$ & $52.1 \%$ & $45.4 \%$ & $58.8 \%$ & $38.7 \%$ & $55.4 \%$ & $38.7 \%$ \\
\hline & & Average & $36.9 \%$ & $69.0 \%$ & $51.9 \%$ & $47.1 \%$ & $56.0 \%$ & $42.8 \%$ & $54.6 \%$ & $45.7 \%$ \\
\hline
\end{tabular}

It gives the minimum distance between any two senses for the two words in the WordNet tree (result normalized to 0-1). If a chromosome encodes scenes (groups of dialogue) $S_{l}$, $S_{2}, \ldots, S_{z}$, with sets of keywords $K_{1}, K_{2}, \ldots, K_{n}$ respectively, then the fitness $F_{\text {wordnet }}$ is given by

$$
F_{\text {wordnet }}=\sum_{i=2}^{n} \sum_{\substack{x \in K_{i}, y \in K_{i-1}}} d(x, y) \text {. }
$$

(c) Combined Effect.

To examine the combined effect of the above two distances we have also experimented with the following fitness function which mixes the effect of both.

$$
F_{\text {combined }}=F_{\text {sentiment }} F_{\text {wordnet }}
$$

\section{B. Evaluation of Solution}

Once the fittest chromosome across all the generations was obtained, the solution was obtained. We have used the Hamming distance for the evaluation purpose as our solutions have been encoded using binary encoding. Hamming distance of two binary strings is the number of bit positions that differ. Since we had prepared our resource files with the sceneboundary information, we were able to construct the actual solution as per our encoding scene. Then we compared the solution obtained using the GA with the former. If $S_{\text {actual }}$ was the binary string representing the actual solution and $S_{G A}$ the solution obtained using GA, we defined the performance metric $\mathrm{P}_{\mathrm{GA}}$ as follows:

$$
\begin{gathered}
P_{G A}=\left(1-\frac{\text { HammingDistance }\left(S_{\text {actual }}, S_{G A}\right)}{\# \text { Dialogueunitsinthescript }-1}\right) \times 100 \% \\
=\frac{\# \text { Bit positions that match between } S_{G A} \& S_{\text {actual }}}{\text { Size of encoding of segmented dialogue sequence }} \times 100 \% .
\end{gathered}
$$

The present task is not a supervised learning problem. So accurate spotting of scene transitions is understandably difficult. Thus, besides counting the number of correct transitions located, we have also tried to evaluate the performance differently. The above metric measures the similarity of the dialogue grouping output by the system with the original scene-wise grouping.
We present a simple baseline for comparison. We do not use identities of speakers (their names) of turns anywhere in the experiments. Instead, word overlap of scenes was used in devising a baseline. Say, $S_{i}$ and $S_{i-1}$ are adjacent dialogue scenes with the sets of words (stop words and named entities excluded) $W_{i}$ and $W_{i-1}$. The word overlap can be given by the Jaccard index:

$$
J\left(S_{i-1}, S_{i}\right)=\frac{\left|W_{i} \cap W_{i-1}\right|}{\left|W_{i} \cup W_{i-1}\right|} .
$$

The closer $J\left(S_{i-1}, S_{i}\right)$ gets to 1 the greater the similarity of adjacent scenes $S_{i}$ and $S_{i-1}$. Put in another way, the closer $J^{\prime}\left(S_{i-1}, S_{i}\right)=1-J\left(S_{i-1}, S_{i}\right)$ gets to 1 greater the dissimilarity of the same scenes in terms of common words. Thus the baseline fitness was devised as follows:

$$
F_{\text {baseline }}=\sum_{i=2}^{n} J^{\prime}\left(S_{i-1}, S_{i}\right)
$$

\section{RESUlTS AND OBSERVATIONS}

A number of scripts have been chosen and processed for resource preparation. Scripts of three movies, namely Godfather-II, Casino and Citizen Kane have been chosen for the purpose of experimentation. These scripts have been picked up due to the presence of abundant dialogue units (in excess of 1000). Table 1 sums up the performance of the scene transition detection algorithm. It reports the proposed performance metric $P_{G A}$ as well as percentage of transitions correctly detected.

When the encoded version of a solution is considered, the proposed $P_{G A}$ reflects the extent of similarity of the obtained solution with the actual one. In other words it reflects a score for the obtained solution.

The results report an average accuracy of $47.1 \%$ in correct detection of scene transitions with the proposed fitness metric $F_{\text {sentiment }}$. Use of Wordnet features in fitness computation improves the average value of $P_{G A}$ but results in a fall in average accuracy to $42.8 \%$. The combined sentiment and Wordnet features produce an average accuracy of $45.7 \%$. It seems that SentiWordNet features have been more informative than WordNet based distance. 
We have observed a lot of false detections of scene boundaries (detection of a transition where the scene should actually continue), resulting in over-fragmentation of dialogue sequence. The modest values for $P_{G A}$ may be attributed to the same. Moreover, no information about the distribution of number of transitions in scripts was available. Thus, the GA used in our experiments initializes each chromosome randomly without a bias to a ' 0 ' (scene continuation) or ' 1 ' (scene transition). There has been no bias in mutating a ' 0 ' or ' 1 ' valued gene.

As far as the baseline is concerned, its average $P_{G A}$ score of $36.9 \%$ is outperformed although it gives higher average scene transition detection accuracy (69.0\%). We observed that in the baseline system the number of scenes output by the system was way higher than that in the original scripts. For example, in Citizen Kane as many as 731 scenes were output while the script describes 88 scenes of dialogue only. In other words, the solution chromosome for all the three movies contain mostly ' 1 's, under the binary encoding scheme. However, $P_{G A}$ dips as expected.

\section{CONCLUSIONS AND FUTURE DiRECTIONS}

In this article, we have reported a mechanism to fragment a sequence of movie script dialogue into scene-wise groups. Wordnet based semantic relatedness and SentiWordnet based affective features have been fed to a genetic algorithm framework to obtain a fragmented, scene-segmented dialogue sequence. The initial results have been encouraging. As a part of future work we are planning to apply our mechanism to easily available movie subtitles. The time information present in subtitles may be capitalized. It may be noted that subtitles hardly contain information about the setting in the movie and conveys mostly the dialogue. The proposed scheme coupled with the time information of subtitles seems to be appropriate for locating scene transitions. Besides, the same algorithm can be suitably modified for identification of emotional episodes in conversation from subtitles or scripts. We are planning to incorporate more textual features (e.g. co-reference resolution) into the proposed framework.

\section{REFERENCES}

[1] J. J. Jung, E. You, and S. B. Park, "Emotion-based character clustering for managing story-based contents: a cinemetric analysis," Multimedia Tools and Applications, pp. 1-17, 2012.

[2] G. I. Lin, and M. A. Walker, "All the world's a stage: Learning character models from film," In Proceedings of the Seventh AI and Interactive Digital Entertainment Conference, AIIDE, vol. 11, 2011.

[3] M. A. Walker, G. I. Lin, and J. E. Sawyer, "An Annotated Corpus of Film Dialogue for Learning and Characterizing Character Style," Proceedings of LREC, 2012.

[4] R. E. Banchs, "Movie-DiC: a movie dialogue corpus for research and development," In Proc. of the 50th Annual Meeting of the ACL. 2012, 2012.

[5] M. Cooper, and J. Foote, "Scene boundary detection via video selfsimilarity analysis," International Conference on Image Processing, vol. 3, pp. 378-381, IEEE, 2001.

[6] J. Wang, and T. S. Chua, "A cinematic-based framework for scene boundary detection in video," The Visual Computer, vol. 19, no.5, 2003, pp-329-341.

[7] T. Cour, C. Jordan, E. Miltsakaki, and B. Taskar, "Movie/script:Alignment and parsing of video and text transcription," Proc. $10^{\text {th }}$ European Conf. Computer Vision, 2008, pp. 158-171.

[8] S. B. Park, H. N. Kim, H. Kim, and G. S. Jo, "Exploiting ScriptSubtitles Alignment to Scene Boundary Detection in Movie," In Multimedia (ISM), 2010 IEEE International Symposium on, pp. 49-56. IEEE (2010)

[9] R. Turetsky, and N. Dimitrova, "Screenplay alignment for closed system speaker identification and analysis of feature films," Proc. IEEE Int. Conf. Multimedia and Expo (ICME'04), 2004, pp. 1659-1662.

[10] D. E. Goldberg, "Genetic Algorithms in Search, Optimization and Machine Learning," Addi-son-Wesley, New York, 1989.

[11] L. (Ed.) Davis, "Handbook of Genetic Algorithms," Van Nostrand Reinhold, New York, 1991.

[12] A. Jhala, "Exploiting Structure and Conventions of Movie Scripts for Information Retrieval and Text Mining," Interactive Storytelling, 2008, pp. 210-213.

[13] B. Stefano, A. Esuli, and F. Sebastiani, "Sentiwordnet 3.0: An enhanced lexical resource for sentiment analysis and opinion mining," In Proceedings of the Seventh conference on International Language Resources and Evaluation (LREC'10), Valletta, Malta, May. European Language Resources Association (ELRA), 2010.

[14] M. C. De Marneffe, C. D. Manning, "Stanford typed dependencies manual," nlp. stan-ford. edu/software/dependencies_manual.pdf, 2008. 